Published in final edited form as:

Lancet Oncol. 2019 January ; 20(1): 31-42. doi:10.1016/S1470-2045(18)30864-7.

\title{
Long-term safety and activity of axicabtagene ciloleucel in refractory large B-cell lymphoma (ZUMA-1): a single-arm, multicentre, phase 1-2 trial
}

\author{
Frederick L Locke*, \\ Moffitt Cancer Center, Tampa, FL, USA \\ Armin Ghobadi, \\ Washington University School of Medicine, St Louis, MO, USA \\ Caron A Jacobson, \\ Dana-Farber Cancer Institute, Boston, MA, USA \\ David B Miklos, \\ Stanford University School of Medicine, Stanford, CA, USA \\ Lazaros J Lekakis, \\ University of Miami Health System, Sylvester Comprehensive Cancer Center, Miami, FL, USA \\ Olalekan O Oluwole, \\ Vanderbilt-Ingram Cancer Center, Nashville, TN, USA
}

Correspondence to: Dr Frederick L Locke, Moffitt Cancer Center, 12902 Magnolia Drive, Tampa, FL 33612, USA frederick.locke@moffitt.org.

Contributors

FLL, JSW, LN, WYG, and SSN designed the study. FLL, AGh, CAJ, DBM, LJL, OOO, YL, IB, BTH, JMT, AD, PMR, PS, IWF, UF, AGo, PAM, JM, TS, JCC, AFH, NLB, WYG, and SSN enrolled and treated patients, and gathered data. FLL, LN, AX, YJ, AB, JMR, JJK, WYG, and SSN analysed and interpreted data. All authors participated in writing the Article, provided feedback throughout the development process, and approved the final submitted version.

* These authors contributed equally

Declaration of interests

FLL has served on scientific advisory boards for Kite and Novartis and reports consultancy fees for Cellular Biomedicine and research support from Forma Therapeutics. AGh has received speaker fees and research funding from, and served on scientific advisory boards for, Kite. CAJ reports personal fees from Kite, Novartis, Precision Bioscience, Bayer, Pfizer, and Humanigen. DBM has received grants and research funding from, and served on scientific advisory boards for, Kite. IB reports speaker fees from Kite. BTH has served on advisory boards for Gilead. AD has served on advisory boards for Kite, Agios, and Novarits, and received research funding from Bristol-Myers Squibb. PMR reports research funding from Seattle Genetics, and has served on advisory boards for, and received personal fees from, Curis. IWF reports research funding from Kite, Agios, ArQule, Beigene, Calithera, Celgene, Constellation, Curis, Forma, Forty-Seven, Genentech, Gilead, Incyte, Infinity, Janssen, Merck, Novartis, Pfizer, Pharmacyclics, Portola, Seattle Genetics, Takeda, TG Therapeutics, Trillium, Verastem, and Roche. UF reports travel support from Kite and honoraria from Celgene. AGo has participated in speakers bureaus for, and received research funding from, Takeda, Kite, Gilead, Pharmacyclics, Janssen, Genentech, and Acerta, and has a leadership role at COTA, a for-profit health-care company. PAM reports speaker and consultant fees from Kite. JM has participated on speakers bureaus or advisory boards for Kite, Pharmacyclics, Janssen, Bayer, Alexion, Pfizer, Juno, Celgene, Bristol-Myers Squibb, Genentech, and Kyowa. TS reports travel support from Kite, speaker and consultancy fees from Pharmacyclics; speaker fees from Janssen and Seattle Genetics, and consultancy for Juno and BeiGene. JCC has participated in advisory boards or speakers bureaus for Kite, Genentech, Novartis, Bayer, and Janssen, and has received research support from Merck. AFH reports grants, personal fees, and consultancy for Bristol-Myers Squibb, Genentech, Merck, Pharamcyclics, and Kite, and grants and consultancy for Gilead. NLB reports research funding from Affimed, Bristol-Meyers Squibb, Celgene, Forty Seven, Genentech, Gilead, Immune Design, Kite, Merck, Millennium, Pharmacyclics, and Acerta, and has served on advisory boards for Pfizer. LN, JMR, AX, YJ, JJK, and WYG, are employed by Kite, and have equity ownership in Gilead. AB and JSW report employment and an issued patent with Kite. SSN reports personal fees and research support from Kite, Merck, and Celgene, research support from BristolMyers Sqibb, Poseida, Cellectis, Karus, and Acerta Pharma, and personal fees from Novartis, Pfizer, and Unum Therapeutics. LJL, OOO, YL, JMT, and PS declare no competing interests. 
Yi Lin,

Mayo Clinic, Rochester, MN, USA

Ira Braunschweig,

Montefiore Medical Center, Albert Einstein College of Medicine, Bronx, NY, USA

Brian T Hill,

Cleveland Clinic Foundation, Cleveland, $\mathrm{OH}, \mathrm{USA}$

John M Timmerman,

UCLA David Geffen School of Medicine, Los Angeles, CA, USA

Abhinav Deol,

Karmanos Cancer Center, Wayne State University, Detroit, MI, USA

Patrick M Reagan,

University of Rochester Medical Center, Rochester, NY, USA

Patrick Stiff,

Loyola University Chicago Stritch School of Medicine, Maywood, IL, USA

lan W Flinn,

Sarah Cannon Research Institute, Nashville, TN, USA

Umar Farooq,

University of lowa, lowa City, IA, USA

Andre Goy,

John Theurer Cancer Center, Hackensack, NJ, USA

Peter A McSweeney,

Colorado Blood Cancer Institute, Denver, CO, USA

Javier Munoz,

Banner MD Anderson Cancer Center, Gilbert, AZ, USA

Tanya Siddiqi,

City of Hope National Medical Center, Duarte, CA, USA

Julio C Chavez,

Moffitt Cancer Center, Tampa, FL, USA

Alex F Herrera,

City of Hope National Medical Center, Duarte, CA, USA

Nancy L Bartlett,

Washington University School of Medicine, St Louis, MO, USA

Jeffrey S Wiezorek,

Kite, Santa Monica, CA, USA

Lynn Navale,

Kite, Santa Monica, CA, USA

Allen Xue, 
Kite, Santa Monica, CA, USA

Yizhou Jiang,

Kite, Santa Monica, CA, USA

Adrian Bot,

Kite, Santa Monica, CA, USA

John M Rossi,

Kite, Santa Monica, CA, USA

Jenny J Kim,

Kite, Santa Monica, CA, USA

William Y Go,

Kite, Santa Monica, CA, USA

Sattva S Neelapu*

University of Texas MD Anderson Cancer Center, Houston, TX, USA

\section{Summary}

Background-Axicabtagene ciloleucel is an autologous anti-CD19 chimeric antigen receptor (CAR) T-cell therapy. In the previous analysis of the ZUMA-1 registrational study, with a median follow-up of 15.4 months (IQR 13.7-17.3), 89 (82\%) of 108 assessable patients with refractory large B-cell lymphoma treated with axicabtagene ciloleucel achieved an objective response, and complete responses were noted in $63(58 \%)$ patients. Here we report long-term activity and safety outcomes of the ZUMA-1 study.

Methods-ZUMA-1 is a single-arm, multicentre, registrational trial at 22 sites in the USA and Israel. Eligible patients were aged 18 years or older, and had histologically confirmed large B-cell lymphoma - including diffuse large B-cell lymphoma, primary mediastinal B-cell lymphoma, and transformed follicular lymphoma-according to the 2008 WHO Classification of Tumors of Hematopoietic and Lymphoid Tissue; refractory disease or relapsed after autologous stem-cell transplantation; an Eastern Cooperative Oncology Group performance status of 0 or 1; and had previously received an anti-CD20 monoclonal antibody containing-regimen and an anthracyclinecontaining chemotherapy. Participants received one dose of axicabtagene ciloleucel on day 0 at a target dose of $2 \times 10^{6} \mathrm{CAR}$ T cells per kg of bodyweight after conditioning chemotherapy with intravenous fludarabine ( $30 \mathrm{mg} / \mathrm{m}^{2}$ body-surface area) and cyclophosphamide $\left(500 \mathrm{mg} / \mathrm{m}^{2}\right.$ bodysurface area) on days $-5,-4$, and -3 . The primary endpoints were safety for phase 1 and the proportion of patients achieving an objective response for phase 2, and key secondary endpoints were overall survival, progression-free survival, and duration of response. Pre-planned activity and safety analyses were done per protocol. ZUMA-1 is registered with ClinicalTrials.gov, number . Although the registrational cohorts are closed, the trial remains open, and recruitment to extension cohorts with alternative endpoints is underway.

Findings-Between May 19, 2015, and Sept 15, 2016, 119 patients were enrolled and 108 received axicabtagene ciloleucel across phases 1 and 2. As of the cutoff date of Aug 11, 2018, 101 patients assessable for activity in phase 2 were followed up for a median of 27.1 months (IQR 25.7-28.8), 84 (83\%) had an objective response, and 59 (58\%) had a complete response. The 
median duration of response was $11 \cdot 1$ months (4-2-not estimable). The median overall survival was not reached (12.8-not estimable), and the median progression-free survival was 5.9 months (95\% CI 3.3-15.0). 52 (48\%) of 108 patients assessable for safety in phases 1 and 2 had grade 3 or worse serious adverse events. Grade 3 or worse cytokine release syndrome occurred in $12(11 \%)$ patients, and grade 3 or worse neurological events in 35 (32\%). Since the previous analysis at 1 year, additional serious adverse events were reported in four patients (grade 3 mental status changes, grade 4 myelodysplastic syndrome, grade 3 lung infection, and two episodes of grade 3 bacteraemia), none of which were judged to be treatment related. Two treatment-related deaths (due to haemophagocytic lymphohistiocytosis and cardiac arrest) were previously reported, but no new treatment-related deaths occurred during the additional follow-up.

Interpretation-These 2-year follow-up data from ZUMA-1 suggest that axicabtagene ciloleucel can induce durable responses and a median overall survival of greater than 2 years, and has a manageable long-term safety profile in patients with relapsed or refractory large B-cell lymphoma.

\section{Introduction}

The prognosis of patients with large B-cell lymphoma refractory to first-line or second-line therapy is poor. ${ }^{1}$ SCHOLAR-1 was an international, retrospective study ${ }^{1}$ in which outcomes were assessed in patients with refractory large B-cell lymphoma, which was defined as progressive or stable disease as best response to first-line or second-line chemotherapy, or relapse less than 12 months after high-dose therapy with haemopoietic stem-cell transplantation. In this population, an estimated 26\% (95\% CI 21-31) of 523 patients had an objective response to standard-of-care therapy, and 7\% (3-15) had a complete response; and median overall survival was $6 \cdot 3$ months $(95 \%$ CI $5 \cdot 9-7 \cdot 0)$.

Chimeric antigen receptor (CAR) T-cell therapies targeting CD19 are a promising approach for the management of refractory large B-cell lymphoma. ${ }^{2-6}$ Axicabtagene ciloleucel is an autologous anti-CD19 CAR T-cell therapy generated by introducing the CAR construct into primary $\mathrm{T}$ cells with a replication-incompetent retroviral vector. ${ }^{7,8}$ The CAR extracellular domain has a singlechain variable fragment targeting CD19, CD28 hinge and transmembrane domains, and intracellular signalling domains that consist of CD3 $\zeta$ and CD28 for T-cell activation and costimulation, respectively. ${ }^{9}$ In the single-arm, multicentre, phase 1-2 ZUMA-1 study ${ }^{10}$ of axicabtagene ciloleucel, at a median follow-up of $15 \cdot 4$ months (IQR 13.7-17·3), 89 (82\%) of 108 assessable patients with refractory large B-cell lymphoma had an objective response, $63(58 \%)$ had a complete response, and $45(42 \%)$ were in remission. ${ }^{10}$ These results led to approval of axicabtagene ciloleucel in the USA and EU for treatment of patients with relapsed or refractory large B-cell lymphomas-including diffuse large B-cell lymphoma, primary mediastinal B-cell lymphoma, high-grade B-cell lymphoma, and transformed follicular lymphoma-as a third-line and higher therapy. ${ }^{11,12}$ Another anti-CD19 CAR T-cell therapy, tisagenlecleucel, was approved for relapsed or refractory diffuse large B-cell lymphoma as a third-line and higher therapy shortly thereafter. 13 There is great clinical interest in the long-term safety of, and durability of responses to, these treatments. In single-centre trials 6,14 with small study populations, durable responses as long as 56 months have been reported with CD28 costimulatory CD19 CAR constructs, and as long as 37.9 months with CD137 (4-1BB) costimulatory CD19 CAR constructs, with 
minimal late toxicities in patients with large B-cell lymphoma. Additionally, other multicentre trials of anti-CD19 CAR- T cell therapy in adult relapsed or refractory large Bcell lymphoma are ongoing.

The general structure of the three CD19 CAR T-cell therapies-axicabatgene ciloleucel, tisagenlecleucel, and lisocabtagene maraleucel-is similar in that all use the same singlechain variable fragment (FMC63) and use CD3 for intracellular signalling. However, they use different combinations of transmembrane and costimulatory domains. Axicabatagene ciloleucel uses CD28 for transmembrane and activation domains, tisagenlecleucel uses CD8 for the transmembrane domain and 4-1BB for costimulation, and lisocabtagene maraleucel uses a CD28 transmembrane domain and 4-1BB for costimulation.

In JULIET, the phase 2 trial of tisagenlecleucel, 48 (52\%) of 93 treated patients with assessable activity achieved an objective response at a median follow-up of 14 months. ${ }^{5}$ Grade 3 or worse cytokine release syndrome occurred in $22 \%$ of 111 patients and neurological events were reported in $12 \% .{ }^{5}$ In an interim analysis of TRANSCEND, the phase 1-2 trial of lisocabtagene maraleucel, with a median follow-up of 8 months, $80 \%$ of 73 patients with either diffuse large B-cell lymphoma not otherwise specified or high-grade B-cell lymphoma, the population selected for the registrational cohort, achieved an objective response. One (1\%) of 73 patients had grade 3 or worse cytokine release syndrome and 11 $(15 \%)$ had neurological events. ${ }^{4}$

Here, we report 2-year safety and activity results from ZUMA-1, and present data on persistence of CAR T cells and recovery of B cells in patients with refractory large B-cell lymphoma and an ongoing response to axicabtagene ciloleucel.

\section{Methods}

\section{Study design and participants}

ZUMA-1 is a, single-arm, multicentre, registrational, phase $1-2$ study 3,10 at 22 medical cancer centres in the USA and Israel (appendix p 2). Eligible patients were aged 18 years or older, and had refractory large B-cell lymphoma-including diffuse large B-cell lymphoma, primary mediastinal B-cell lymphoma, and transformed follicular lymphoma-according to the 2008 WHO Classification of Tumours of Haematopoietic and Lymphoid Tissue that was histologically confirmed retrospectively by independent pathology review; ${ }^{15}$ an Eastern Cooperative Oncology Group performance status of 0 or 1 ; an absolute neutrophil count of at least 1000 per $\mu \mathrm{L}$; a platelet count of at least 75000 per $\mu \mathrm{L}$; and adequate organ function. 3,10 Refractory disease was defined as progressive or stable disease as best response to the most recent chemotherapy regimen, or disease progression or relapse within 12 months of autologous stem-cell transplantation. Patients also needed to have previously received a regimen containing an anti-CD20 monoclonal antibody and an anthracycline-containing chemotherapy regimen. Patients with transformed diffuse large B-cell lymphoma must have received previous chemotherapy for follicular lymphoma and developed chemorefractory disease after transformation. Patients who had undergone autologous stem-cell transplantation within 6 weeks of informed consent for ZUMA-1, or those who had previously undergone allogeneic haemopoietic stem-cell transplantation, or received 
previous CD19-targeted therapy or CAR T-cell therapy were excluded. ${ }^{3,10}$ The full eligibility criteria are listed in the protocol (appendix).

Each study site's institutional review board reviewed and approved the study protocol and amendments, and all patients provided written informed consent. The study was done according to the International Conference on Harmonisation Good Clinical Practice guidelines. An independent data safety monitoring board reviewed safety and activity data during the phase 2 registrational portion of the study when 20 and 50 patients in the diffuse large cell B-cell lymphoma cohort had the opportunity to complete the 3-month disease assessment.

\section{Procedures}

Eligible patients underwent leukapheresis to obtain peripheral blood mononuclear cells for CAR T-cell production. After manufacture of the $\mathrm{T}$ cells, patients received conditioning chemotherapy consisting of intravenous fludarabine $\left(30 \mathrm{mg} / \mathrm{m}^{2}\right.$ body-surface area per day) and cyclophosphamide ( $500 \mathrm{mg} / \mathrm{m}^{2}$ body-surface area per day) on days $-5,-4$, and -3 , before receiving a single intravenous infusion of axicabtagene ciloleucel on day 0 at a target dose of $2 \times 10^{6} \mathrm{CAR} \mathrm{T}$ cells per $\mathrm{kg}$ of body-weight. ${ }^{3,10}$ As per protocol, bridging chemotherapy was not permitted between leukapheresis and conditioning chemotherapy. Patients could be removed from the study if they withdrew consent for further follow-up, were lost to follow-up, or died.

Laboratory monitoring was done within approximately 5 days of enrolment, on days $-5,-4$, and -3 , day 0 (ie, the day of axicabtagene ciloleucel infusion), within the first 7 days of infusion, at week 2, month 1 , month 2, month 3, and then every 3 months until month 24 . Response was assessed locally by PET according to the International Working Group Response Criteria for Malignant Lymphoma ${ }^{10,16}$ at month 1, every three 3 months from month 3 to month 24, and as clinically indicated or per the institution's standard-of-care after 2 years. For phase 2, disease response was also assessed by an independent central review committee.

All observed adverse events were monitored continuously from enrolment until month 3 after treatment with axicabtagene ciloleucel infusion. After 3 months, only targeted adverse events and serious adverse events in the categories of neurological events, haematological events, infections, autoimmune disorders, and secondary malignancies were reported until 24 months or disease progression. Prolonged cytopenias are an emerging class-effect of CAR T cells, ${ }^{17}$ and therefore we assessed the frequency of grade 3 or worse cytopenias at month 3 or later. Cytokine release syndrome was graded with the modified criteria of Lee and colleagues. ${ }^{18}$ Individual symptoms of cytokine release syndrome, all other adverse events, and baseline laboratory values were graded according to the National Cancer Institute's Common Terminology Criteria for Adverse Events (version 4.03). ${ }^{19}$

CAR T-cell concentrations in the blood were assessed by PCR analysis enumerating CAR gene-marked cells, as previously described. ${ }^{3}$ Additionally, post-hoc analyses of responses were done by cell of origin and in patients with double-expressor or high-grade B-cell 
lymphoma, progression-free survival by response status at month 3 , and B-cell recovery in patients with ongoing responses (appendix p 3).

\section{Outcomes}

The primary endpoint of phase 1 was the incidence of dose-limiting toxicities, as previously reported. ${ }^{3}$ The primary endpoint of phase 2 was the proportion of patients achieving an objective response (ie, the combined proportion of participants who had a complete or partial response) as per investigators' assessment.

Secondary endpoints were overall response by independent-review committee assessment, duration of response (defined as the time from the first objective response to disease progression or death from any cause), progression-free survival (defined as the time from infusion to the date of disease progression or death from any cause), overall survival (defined as the time from infusion to the date of death from any cause), incidence of adverse events, and concentrations of anti-CD19 CAR T cells in blood, anti-axicabtagene ciloleucel antibodies, and concentrations of cytokines in serum. Concentrations of anti-axicabtagene ciloleucel antibodies in blood and cytokines in serum have been previously reported. ${ }^{10}$

\section{Statistical analysis}

The previously reported primary analysis (cutoff Jan 27, 2017) ${ }^{10}$ was done when 92 patients could be assessed for response, 6 months after the axicabtagene ciloleucel infusion, to differentiate between an active therapy with a $40 \%$ true response rate and a therapy with a response rate of $20 \%$ or less, with a power of at least $90 \%$ and a one-sided a of 0.025 .

The data cutoff date for the current analyses was Aug 11, 2018. As per protocol, inferential testing was done only for the primary endpoint assessing activity in patients enrolled in phase 2 who received axicabtagene ciloleucel. Incidence of adverse events in all treated patients and changes in laboratory values were tabulated and summarised with descriptive statistics.

Activity analyses excluded patients from phase 1, in whom activity was not assessed by the Independent Central Review Committee. The safety analysis included all patients who received axicabtagene ciloleucel in both phases 1 and 2 .

The proportion of patients with objective responses was summarised with descriptive statistics. Time-to-event analyses of duration of response, progression-free survival, and overall survival were assessed with the Kaplan-Meier method, and 95\% CIs for responses were calculated with the Clopper-Pearson method.

For survival analyses, patients who had not died were censored at the last date known alive. For progression-free survival and duration of response, patients who had not died or experienced disease progression were censored at their last evaluable disease assessment or date of stem-cell transplantation. Concordance between activity assessments by investigators and the independent central review committee review was measured as the proportion of patients for whom central assessment of objective or complete response matched investigator assessments. 
All statistical analyses were done in SAS (version 9.4). This trial is registered with ClinicalTrials.gov, number .

\section{Role of the funding source}

The study funder participated in the study design; data collection, analysis, and interpretation; and writing of the report. All authors had full access to all study data and the corresponding author had final responsibility for the decision to submit for publication.

\section{Results}

Between May 19, 2015, and Sept 15, 2016, 119 patients were enrolled and 108 received axicabtagene ciloleucel (seven in phase 1 and 101 in phase 2; appendix p 17). Patient characteristics are listed in table $1.52(70 \%)$ of 74 patients assessed for cell of origin had germinal centre B-cell-like disease and 18 (24\%) had activated B-cell-like disease. Of the 47 patients with pretreatment tumour samples, 30 (64\%) had double expressor B-cell lymphoma and seven (15\%) had high-grade B-cell lymphoma, including one (2\%) with triple-hit high-grade B-cell lymphoma, four (9\%) with double-hit high-grade B-cell lymphoma, and two (4\%) with high-grade B-cell lymphoma not otherwise specified (appendix p 5).

As of Aug 11, 2018, 101 patients assessable for activity in phase 2 were followed up for a median of 27.1 months (IQR 25.7-28.8). According to investigator assessment, 84 (83\%) of 101 patients had an objective response to axicabtagene ciloleucel-59 (58\%) complete responses and 25 (25\%) partial responses (table 2; appendix p 6). Ten (10\%) patients had stable disease, five (5\%) had progressive disease as best response, and two (2\%) could not be assessed. Concordance between investigator assessments and Independent Central Review Committee assessments was $81 \%$ for objective response and $90 \%$ for complete response (table 2). Median time to response was 1 month (IQR 1-1). ${ }^{10} 11$ of 33 patients with partial responses at 1 month, and 11 of 24 patients with stable disease at 1 month, subsequently achieved a complete response, with most conversions occurring by 6 months (appendix $\mathrm{p}$ 18). Among the 33 patients with double-expressor and high-grade B-cell lymphoma, 30 (91\%) achieved an objective response and $23(70 \%)$ achieved a complete response by investigator assessment (appendix p 7). The median duration of response for all 101 patients was 11.1 months (95\% CI 4-2-not estimable) by investigator assessment (figure 1A; table 2) and was not reached by independent central review committee review (appendix p 19). The median duration of response for participants with complete responses was not reached (95\% CI 12.9-not estimable). 39 (39\%) patients had ongoing responses and were censored at the last disease assessment before the data cutoff, including 37 (37\%) with ongoing complete responses (table 2). Only two (5\%) of the 39 patients with ongoing responses underwent allogeneic stem-cell transplantation, and none underwent autologous stem-cell transplantation while in axicabtagene-ciloleucel-induced remission. Ongoing responses were consistent across key baseline and clinical covariates (appendix p 20).

By investigator assessment, 61 of 101 patients had disease progression or died on study. Median progression-free survival was 5.9 months (95\% CI 3.3-15.0; figure 1B). In post-hoc analyses, the estimated proportion of patients with progression-free survival at 24 months 
was $72.0 \%$ (95\% CI 56-0-83.0) among those with complete responses at 3 months, 75.0\% (31.5-93.1) among those with partial responses at 3 months, and 22.2\% (3.4-51.3) among those with stable disease at 3 months (figure 2). The median overall survival was not reached (95\% CI 12.8-not estimable; figure 1C), with an estimated 24-month survival proportion of $50.5 \%$ (95\% CI 40.2-59.7). No patients were lost to follow-up.

Consistent with previously reported results for objective response, ${ }^{10}$ ongoing response at 24 months was associated with higher CAR T-cell peak concentrations and area under the curve in the first 28 days after axicabtagene ciloleucel infusion (figure 3). By 24 months, 11 (34\%) of 32 assessable patients maintained ongoing responses but no longer had detectable genemarked CAR T cells (appendix p 21).

The safety profile of axicabtagene ciloleucel 2 years after infusion was largely similar to that in previous reports. ${ }^{3,10}$ All 108 treated patients had adverse events, and 106 (98\%) had grade 3 or worse adverse events (table 3; appendix pp 8-14). 52 (48\%) of 108 patients had grade 3 or worse serious adverse events (appendix p 8). Grade 3 or worse cytokine release syndrome occurred in $12(11 \%)$ patients and grade 3 or worse neurological events occurred in 35 (32\%); all these events were manageable and largely reversible (appendix p 8). Since the 12month analysis (data cutoff Aug 11, 2017), no new cases of cytokine release syndrome or neurological events related to axicabtagene ciloleucel were reported.

30 (28\%) patients experienced grade 3 or worse infections during the study. Since the 12month analysis, four patients developed new, serious adverse events, none of which were related to axicabtagene ciloleucel (appendix p 15). One patient had grade 3 mental status changes at 15.6 months, which were attributed to vasovagal episode in the context of hypovolaemia and resolved within 6 days. One patient developed a grade 3 lung infection at month 19.3, which resolved within 36 days. Another had two episodes of grade 3 bacteraemia (at 15.5 months and 20.7 months), both of which resolved within 6 days. The fourth patient developed grade 4 myelodysplastic syndrome at 18.9 months, which was attributed to previous chemotherapy that was ongoing at data cutoff.

$18(17 \%)$ of 108 patients had grade 3 or worse cytopenias at 3 months or later, including 12 (11\%) with neutropenia, eight (7\%) with thrombocytopenia, and three (3\%) with anaemia. At the time of analysis, two patients had grade 3 cytopenias (one anaemia, one neutropenia; appendix p 16). 3 months after axicabtagene ciloleucel infusion, six (17\%) of the 35 assessable patients with ongoing responses had detectable B cells in peripheral blood. At 9 months, 20 (61\%) of 33 assessable patients had detectable B cells, and, at 24 months, 24 (75\%) of 32 assessable patients had detectable B cells (figure 3). As previously reported, nine $(8 \%)$ of 108 patients received intravenous immunoglobulin therapy between axicabtagene ciloleucel infusion and the day of hospital discharge for first hospitalisation. ${ }^{20}$ Cumulatively, 33 (31\%) of 108 patients received intravenous immunoglobulin therapy, per treating physicians' discretion, including 17 (44\%) of the 39 patients with ongoing responses.

54 (50\%) of 108 patients who received treatment have died since study initiation (four patients in phase 1 and 50 in phase 2). 50 patients have died from progressive disease. Six of 
these deaths occurred after the 12-month analysis. No new axicabtagene ciloleucel-related deaths occurred since the 12-month analysis, but, as previously reported, four adverse-eventrelated deaths occurred during phases 1 and 2 of the study, two of which were related to axicabtagene ciloleucel (appendix p 17). ${ }^{3,10}$ We noted no cases of replication-competent retroviruses or axicabtagene-ciloleucel-related secondary cancers as of this data cutoff.

\section{Discussion}

To our knowledge, this long-term analysis of ZUMA-1 represents the longest follow-up of a large, registrational study of anti-CD19 CAR T-cell therapy. A substantial proportion of patients with refractory large B-cell lymphoma treated with a single infusion of axicabtagene ciloleucel achieved durable responses lasting more than 2 years and needed no further consolidation therapy. Additionally, in this population of patients refractory to several lines of treatment, which included a large proportion of patents with activated B-cell-like, double expressor, and high-grade B-cell lymphoma, outcomes were similar across all patient subgroups. Median overall survival was not yet reached at 2 years, with an estimated 24month survival proportion of 50.5\% (95\% CI 40.2-59.7). This finding represents a major improvement in clinical outcomes for these patients, for whom the expected median overall survival with conventional therapies is approximately 6 months, with a 2-year overall survival of approximately $20 \% .^{1}$ These estimates build on two single-centre trials ${ }^{6,14}$ of antiCD19 CAR T cells that previously showed durable remissions without substantial late toxicities in a total of ten patients with diffuse large B-cell lymphoma.

Axicabtagene ciloleucel had a manageable 2-year safety profile, and late-onset serious adverse events were uncommon. Importantly, despite targeting of CD19 and the expected induction of B-cell aplasia, the frequency of late-onset grade 3 or worse serious infections was low. In fact, $75 \%$ of assessable patients with ongoing responses showed evidence of Bcell recovery by 24 months, and initiation of B-cell recovery was noted in some patients at 9 months. That these patients with ongoing responses recovered B cells suggests the possibility that durable responses in adults with lymphoma do not require longterm persistence of functional CAR T cells. These results accord with National Cancer Institute data showing that three of four patients with long-term ongoing responses had B-cell recovery. ${ }^{14}$ Additional exploration of the importance of tumour-reactive CAR T-cell persistence and its association with B-cell recovery is warranted.

Less than $20 \%$ of patients had grade 3 or worse cytopenias 3 months after treatment. The pathophysiology underlying prolonged cytopenias is unclear. However, they do not seem to place patients at a major risk of late-onset complications. Importantly, none of these patients had clinical sequelae such as major bleeding or needed allogeneic stem-cell transplantation to correct cytopenias. Similar sustained cytopenias have been noted with other anti-CD19 CAR T-cell therapies, ${ }^{13,17,21,22}$ suggesting a possible class effect.

The results of this updated analysis show continuity of the progression-free survival data at 6 months. ${ }^{10}$ With ten additional relapses occurring after 6 months, axicabtagene ciloleucel might offer long-term benefits for a substantial proportion of patients with refractory large B-cell lymphoma. ${ }^{23}$ Additionally, analysis of progression-free survival by response at 3 
months suggests that achievement of complete or partial responses at 3 months might be predictive of long-term response durability. Results from large retrospective studies of lymphoma survivorship databases suggest that sustained responses 24 months after chemoimmunotherapy were associated with improved overall survival at 5 years in patients with diffuse large B-cell lymphoma who previously received first-line treatment or after autologous stem-cell transplantation for relapsed or refractory disease. ${ }^{24-26}$ Myers and colleagues $^{25}$ reported that, among patients with diffuse large B-cell lymphoma who were progression free 24 months after autologous stem-cell transplantation, 5-year overall survival was $89 \%$ (95\% CI 87-91).

Although allogeneic stem-cell transplantation could offer a potential chance of improved long-term overall survival in patients with relapsed or refractory diffuse large B-cell lymphoma, less than a fifth of patients who relapse after autologous stem-cell transplantation are able to undergo allogeneic stem-cell transplantation. ${ }^{27}$ In the randomised, phase 3 CORAL study comparing induction therapy versus rituximab, ifosfamide, carboplatin, and etoposide (R-ICE) with rituximab, dexa-methasone, cytarabine, and cisplatin (RDHAP) before autologous stem-cell transplantation in relapsed or refractory diffuse large Bcell lymphoma, only 21 (5\%) of the 458 enrolled patients underwent allogeneic stem-cell transplantation. ${ }^{28,29}$ A major difference between allogeneic stem-cell transplantation and axicabtagene ciloleucel is that allogeneic stem-cell transplantation is typically used as consolidation therapy for relapsed or refractory diffuse large B-cell lymphoma only after response to salvage chemotherapy. In a large series of patients receiving allogeneic stem-cell transplantation after relapse following autologous stem-cell transplantation, those with chemorefractory disease before allogeneic stem-cell transplantation had a 2-year progression-free survival of approximately $20 \%$ compared with approximately $40 \%$ for those who had chemosensitive disease. ${ }^{30}$ Furthermore, many patients with refractory disease are not candidates for allogeneic stem-cell transplantation because of poor response to salvage therapy, the absence of a donor, older age, or comorbidities. Patients who are candidates for the procedure often experience kinetic failure before the onset of graft-versuslymphoma effects.

By contrast, no upper age limit has yet been defined for treatment with axicabtagene ciloleucel and other CAR T-cell therapies. In ZUMA-1, 25\% of patients were aged 65 years or older, and activity did not seem to have been affected by age. Additionally, many patients enrolled in ZUMA-1 were not eligible for allogeneic stem-cell transplantation because they did not respond to their last previous therapy. Furthermore, since axicabtagene ciloleucel approval, many patients who would not have met the strict eligibility criteria for ZUMA-1, but who were otherwise eligible according to the label, have received axicabtagene ciloleucel therapy as standard of care. Indeed, in a large retrospective study ${ }^{31}$ of more than 150 patients treated with axicabtagene ciloleucel at centres across the USA, short-term safety and activity were similar to those in ZUMA-1 despite nearly half the patients not meeting ZUMA-1 eligibility criteria in terms of performance status, cytopenias, comorbidities, or organ dysfunction, among others. Collectively, these results suggest that axicabtagene ciloleucel CAR T-cell therapy is a feasible treatment option for most patients with relapsed or refractory large B-cell lymphoma. Additionally, the potential benefits of CAR T-cell therapy earlier in the lymphoma disease course are being investigated in 
ZUMA-7, a randomised, international, phase 3 study of the activity and safety of axicabtagene ciloleucel versus standard-of-care second-line treatment for patients with relapsed or refractory diffuse large B-cell lymphoma ().

In addition to axicabtagene ciloleucel, two other anti-CD19 CAR T-cell therapies have either been approved or are under investigation for relapsed or refractory large B-cell lymphoma. Tisagenlecleucel, which has been approved in the USA and EU, is an autologous anti-CD19 CAR T-cell therapy but uses a CD137 (4-1BB) costimulatory domain for T-cell activation, as opposed to CD28, which is used by axicabtagene ciloleucel. ${ }^{5,13}$ In the phase 2 JULIET study (), 111 patients received tisagenlecleucel. With a median follow-up of 14 months, 48 (52\%) of the 93 patients with assessable activity had objective responses and $40 \%$ had complete responses. ${ }^{5}$ Median progression-free survival was 2.9 months (95\% CI 2.2-4.2) and median overall survival was 11.7 months (6.6 to not assessable). TRANSCEND () is a phase 1-2 trial of lisocabtagene maraleucel. Like tisagenlecleucel, lisocabtagene maraleucel includes a CD137 (4-1BB) costi-mulatory domain. ${ }^{4}$ With a median of 8 months of followup, in interim analysis of the phase 1 section of the trial, $80 \%$ of 73 patients treated who had the disease histologies selected for the registrational cohort achieved an objective response, and duration of response was not reached. ${ }^{4}$ Overall survival has not yet been reported, but the frequency of objective responses at 6 months was $47 \%$ (95\% CI 35-59), ${ }^{4}$ which is similar to that in ZUMA-1. Additional follow-up will establish if the responses to tisagenlecleucel and lisocabtagene maraleucel are durable. Although varying toxicity grading scales were used in ZUMA-1, JULIET, and TRANSCEND, grade 3 or worse cytokine release syndrome and neurological events have been recorded in all three trials. In JULIET, grade 3 or worse cytokine release syndrome occurred in $22 \%$ of 111 patients, and neurological events occurred in $12 \%$ of patients, with lower proportions of both (one [1\%] and $11[15 \%]$ of 73 patients, respectively) reported in TRANSCEND. ${ }^{4,5}$

Cross-trial comparisons of efficacy, toxicity, and durability of these second generation antiCD19 CAR T-cell therapies are difficult in view of the small numbers of patients treated in each study, differences in patient populations, baseline characteristics, follow-up times, and methods. Partly due to differences in study design, the proportions of patients who ultimately received CAR T-cell therapy relative to the number enrolled in the trial differed substantially between ZUMA-1, JULIET, and TRANSCEND, and could be relevant when considering the feasibility of therapeutic administration outside trial settings. $108(91 \%)$ of 119 patients enrolled in ZUMA-1 received study therapy, compared with 111 (67\%) of 165 enrolled in JULIET and 114 (85\%) of 134 enrolled in TRANSCEND. ${ }^{4,5}$ Furthermore, bridging chemotherapy after apheresis was allowed in both JULIET and TRANSCEND, which further compounds comparisons with ZUMA-1 and obscures the treatment effect of tisagenlecleucel or lisocabtagene maraleucel in isolation. Additional studies are needed to establish whether there are true differences in the activity and safety between the three CAR T-cell products.

This study has several limitations. The study was designed to show an improvement in response. Additional studies in other large B-cell lymphoma settings are warranted to understand the benefits of axicabtagene ciloleucel in additional settings. Furthermore, we did not address the effect of axicabtagene ciloleucel on quality of life. Additional studies in 
large patient populations are needed to identify factors that could be associated with the risk of prolonged cytopenias with CAR T-cell therapy. Analysis of immunoglobulin concentrations would further elucidate the degree of functional immune recovery after axicabtagene ciloleucel treatment. However, data for immunoglobulin concentrations were not available because collection of immunoglobulin data was per investigator decision and not a protocol-specified assessment. Finally, mechanisms of relapse require further comprehensive analyses and additional studies.

This 2-year analysis of the multicentre ZUMA-1 trial shows that a substantial proportion of patients with refractory large B-cell lymphoma treated with axicabtagene ciloleucel can achieve durable responses with manageable long-term safety. Additional studies are needed to confirm the overall survival results noted here and to establish whether axicabtagene ciloleucel can improve quality of life in this setting. Further research will also help to elucidate the mechanisms of resistance to this therapy.

\section{Supplementary Material}

Refer to Web version on PubMed Central for supplementary material.

\section{Acknowledgments}

This study was funded by Kite, which provided all the study materials, and the Leukemia \& Lymphoma Society Therapy Acceleration Program. We thank the patients who participated in the study, their families, friends, and caregivers, and the study staff and health-care providers at all the clinical trial sites. Medical writing support was provided by Christopher Waldapfel (Kite, Santa Monica, CA, USA), and Skye Geherin (Nexus Global Group Science, IL, USA), which was funded by Kite.

Funding Kite and the Leukemia \& Lymphoma Society Therapy Acceleration Program.

\section{References}

1. Crump M, Neelapu SS, Farooq U, et al. Outcomes in refractory diffuse large B-cell lymphoma: results from the international SCHOLAR-1 study. Blood 2017; 130: 1800-08. [PubMed: 28774879]

2. Brudno JN, Kochenderfer JN. Chimeric antigen receptor T-cell therapies for lymphoma. Nat Rev Clin Oncol 2018; 15: 31-46. [PubMed: 28857075]

3. Locke FL, Neelapu SS, Bartlett NL, et al. Phase 1 results of ZUMA-1: a multicenter study of KTEC19 anti-CD19 CAR T cell therapy in refractory aggressive lymphoma. Mol Ther 2017; 25: 28595. [PubMed: 28129122]

4. Abramson JS, Gordon LI, Palomba ML, et al. Updated safety and long term clinical outcomes in TRANSCEND NHL 001, pivotal trial of lisocabtagene maraleucel (JCAR017) in R/R aggressive NHL. J Clin Oncol 2018; 36 (suppl): abstr 7505.

5. Borchmann P, Tam CS, Jaäger U, et al. An updated analysis of Juliet, a global pivotal phase 2 trial of tisagenlecleucel in adult patients with relapsed or refractory $(\mathrm{R} / \mathrm{R})$ diffuse large B-cell lymphoma (DLBCL). 23rd Congress of the European Hematology Association; Stockholm, Sweden; June 1417, 2018; S799.

6. Schuster SJ, Svoboda J, Chong EA, et al. Chimeric antigen receptor T Cells in refractory B-cell lymphomas. N Engl J Med 2017; 377: 2545-54. [PubMed: 29226764]

7. Kochenderfer JN, Feldman SA, Zhao Y, et al. Construction and preclinical evaluation of an antiCD19 chimeric antigen receptor. J Immunother 2009; 32: 689-702. [PubMed: 19561539]

8. Kochenderfer JN, Yu Z, Frasheri D, Restifo NP, Rosenberg SA. Adoptive transfer of syngeneic T cells transduced with a chimeric antigen receptor that recognizes murine CD19 can eradicate lymphoma and normal B cells. Blood 2010; 116: 3875-86. [PubMed: 20631379] 
9. Roberts ZJ, Better M, Bot A, Roberts MR, Ribas A. Axicabtagene ciloleucel, a first-in-class CAR T cell therapy for aggressive NHL. Leuk Lymphoma 2018; 59: 1785-96. [PubMed: 29058502]

10. Neelapu SS, Locke FL, Bartlett NL, et al. Axicabtagene ciloleucel CAR T-cell therapy in refractory large B-cell lymphoma. N Engl J Med 2017; 377: 2531-4. [PubMed: 29226797]

11. Kite. Yescarta (axicabtagene ciloleucel) [package insert]. https://www.yescarta.com/files/yescartapi.pdf (accessed Nov 20, 2018).

12. Kite. Yescarta (axicbatagene ciloleucel) summary of product characteristics. Amsterdam: Kite, 2018 https://www.medicines.org.uk/emc/product/9439/smpc (accessed Nov 20, 2018).

13. Novartis. Kymriah (tisagenlecleucel) package insert. https://www.fda.gov/downloads/ UCM573941.pdf (accessed Nov 20, 2018).

14. Kochenderfer JN, Somerville RPT, Lu T, et al. Long-duration complete remissions of diffuse large B cell lymphoma after anti-CD19 chimeric antigen receptor T cell therapy. Mol Ther 2017; 25: 2245-53. [PubMed: 28803861]

15. WHO. WHO classification of tumours of haematopoietic and lymphoid tissue, 4th edn. Geneva: World Health Organization, 2008.

16. Cheson BD, Pfistner B, Juweid ME, et al. Revised response criteria for malignant lymphoma. J Clin Oncol 2007; 25: 579-86. [PubMed: 17242396]

17. Brudno JN, Kochenderfer JN. Toxicities of chimeric antigen receptor T cells: recognition and management. Blood 2016; 127:3321-30. [PubMed: 27207799]

18. Lee DW, Gardner R, Porter DL, et al. Current concepts in the diagnosis and management of cytokine release syndrome. Blood 2014; 124: 188-95. [PubMed: 24876563]

19. US Department of Health and Human Services. Common Terminology Criteria for Adverse Events version 4.03. Bethesda, MD: National Institutes of Health, 2010.

20. Neelapu SS, Locke FL, Go WY. Reply to letter to the editor: CAR T-Cell therapy in large B-cell lymphoma. N Engl J Med 2018; 378: 1065.

21. Lee DW, Kochenderfer JN, Stetler-Stevenson M, et al. T cells expressing CD19 chimeric antigen receptors for acute lymphoblastic leukaemia in children and young adults: a phase 1 doseescalation trial. Lancet 2015; 385: 517-28. [PubMed: 25319501]

22. Maude SL, Laetsch TW, Buechner J, et al. Tisagenlecleucel in children and young adults with Bcell lymphoblastic leukemia. N Engl J Med 2018; 378: 439-8. [PubMed: 29385370]

23. Chavez JC, Locke FL. A possible cure for refractory DLBCL: CARs are headed in the right direction. Mol Ther 2017; 25: 2241-43. [PubMed: 28941574]

24. Majhail NS, Bajorunaite R, Lazarus HM, et al. Long-term survival and late relapse in 2-year survivors of autologous haematopoietic cell transplantation for Hodgkin and non-Hodgkin lymphoma. Br J Haematol 2009; 147: 129-39. [PubMed: 19573079]

25. Myers RM, Hill BT, Shaw BE, et al. Long-term outcomes among 2-year survivors of autologous hematopoietic cell transplantation for Hodgkin and diffuse large B-cell lymphoma. Cancer 2018; 124: 816-25. [PubMed: 29125192]

26. Maurer MJ, Ghesquieres H, Jais JP, et al. Event-free survival at 24 months is a robust end point for disease-related outcome in diffuse large B-cell lymphoma treated with immunochemotherapy. J Clin Oncol 2014; 32: 1066-73. [PubMed: 24550425]

27. Rigacci L, Puccini B, Dodero A, et al. Allogeneic hematopoietic stem cell transplantation in patients with diffuse large B cell lymphoma relapsed after autologous stem cell transplantation: a GITMO study. Ann Hematol 2012; 91: 931-39. [PubMed: 22245922]

28. van Den Neste E, Schmitz N, Mounier N, et al. Outcomes of diffuse large B-cell lymphoma patients relapsing after autologous stem cell transplantation: an analysis of patients included in the CORAL study. Bone Marrow Transplant 2017; 52: 216-21. [PubMed: 27643872]

29. van Den Neste E, Schmitz N, Mounier N, et al. Outcome of patients with relapsed diffuse large Bcell lymphoma who fail second-line salvage regimens in the international CORAL study. Bone Marrow Transplant 2016; 51: 51-57. [PubMed: 26367239]

30. Van Kampen RJ, Canals C, Schouten HC, et al. Allogeneic stem-cell transplantation as salvage therapy for patients with diffuse large B-cell non-Hodgkin's lymphoma relapsing after an autologous stem-cell transplantation: an analysis of the European Group for Blood and Marrow Transplantation Registry. J Clin Oncol 2011; 29: 1342-8. [PubMed: 21321299] 
31. Nastoupil LJ, Jain MD, Spiegel Y, et al. Axicabtagene ciloleucel (axi-cel) CD19 chimeric antigen receptor (CAR) T-cell therapy for relapsed/refractory large B-cell lymphoma: real world experience. Blood 2018; 132 (suppl 1): 91. 


\section{Research in context}

\section{Evidence before this study}

We searched PubMed with the terms "CD19 chimeric antigen receptor" AND "lymphoma" AND ("clinical trial" OR "longterm remission") NOT "review"for clinical trials done in humans published in English up to Oct 1, 2018. Evidence of long-term durable responses after anti-CD19 chimeric antigen receptor (CAR)T-cell therapy for high-grade B-cell lymphomas was scarce. We identified 11 citations that included response data for patients with diffuse large B-cell lymphoma, primary mediastinal B-cell lymphoma, or transformed follicular lymphoma who were assessed for outcomes after treatment with anti-CD19 CART-cell therapy. Of these 11 citations, two included outcomes for anti-CD19 CART-cell therapy in conjunction with autologous stem-cell transplantation as a consolidation rather than as primary therapy for refractory diffuse large B-cell lymphoma, two included outcomes after treatment of B-cell malignancies with allogeneic donor-derived anti-CD19 CART cells after relapse post allogeneic stemcell transplantation, and three were previous reports of the JULIET, TRANSCEND and ZUMA-1 trials assessing anti-CD19 CART cells as a salvage treatment for refractory patients with large B-cell lymphoma. The remaining four citations were small phase 1 studies or case series.

\section{Added value of this study}

There is an unmet need for therapies that can increase the proportion of patients with refractory large B-cell lymphoma who can achieve long-term responses. Our results show durable remissions, with ongoing responses in 39 (39\%) of 101 patients who received axicabtagene ciloleucel. No late serious adverse events were attributed to axicabtagene ciloleucel. To our knowledge, ours is the first long-term analysis with more than 2 years of follow-up of a multicentre trial of anti-CD19 CAR T-cell therapy in patients with refractory large B-cell lymphoma. Axicabtagene ciloleucel can offer durable remissions to patients who otherwise lack treatment options.

\section{Implications of all the available evidence}

Our results showing durable responses lasting more than 2 years, combined with the available literature on long-term outcomes with conventional therapies in patients with diffuse large B-cell lymphoma, suggest that axicabtagene ciloleucel could potentially offer long-term responses for a substantial proportion of patients with refractory large Bcell lymphoma who otherwise are at high risk of disease progression with conventional chemotherapy. Axicabtagene ciloleucel had a manageable long-term safety profile, and late serious adverse events were rare. Other investigational and approved CD19 CAR Tcell therapies are available to patients and have shown similar anticancer activity, although with less follow-up. Additional follow-up and further studies are needed to fully characterise the differences in efficacy and safety between CAR T-cell therapies. 


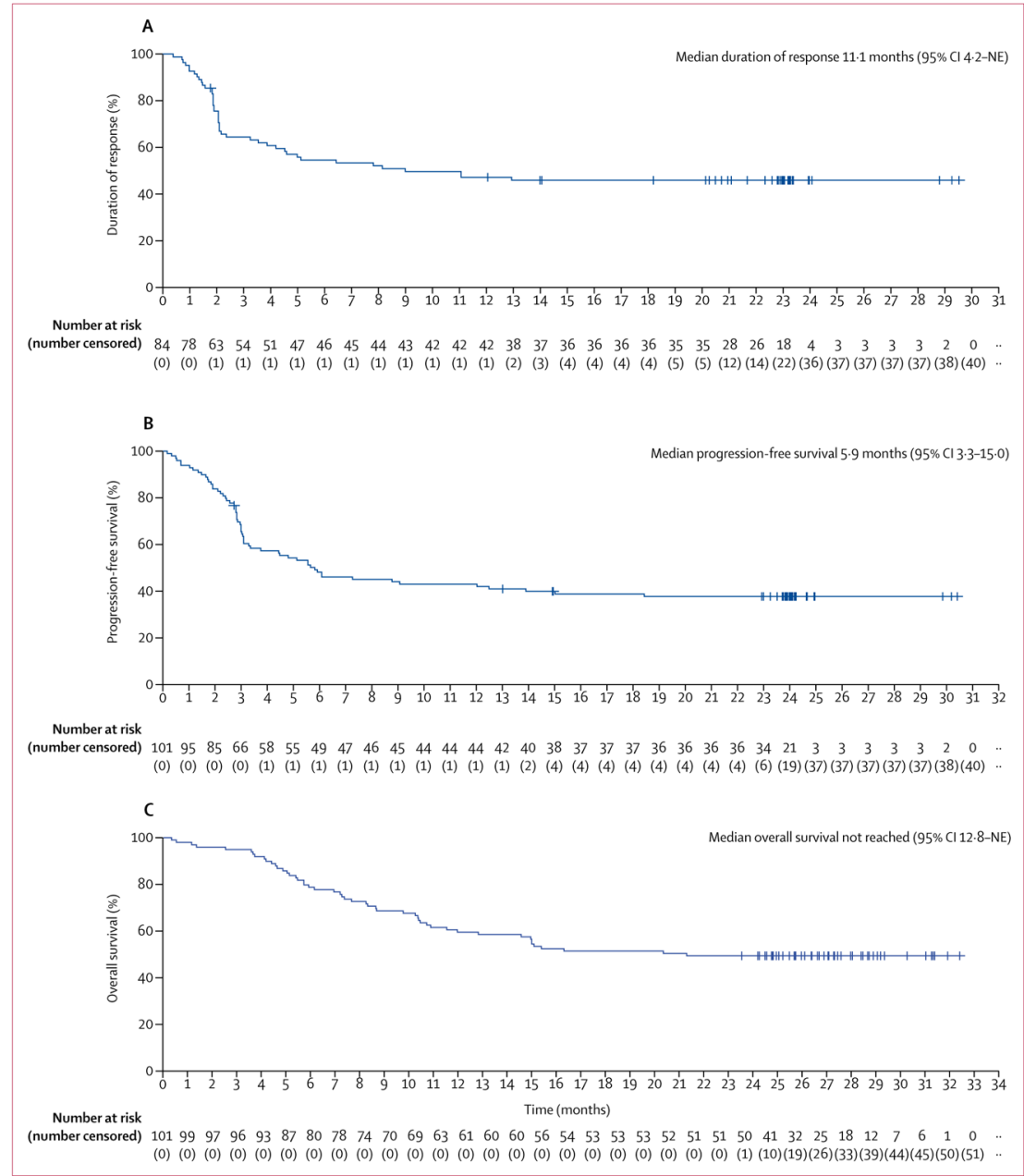

Figure 1: Kaplan-Meier estimates of investigator-assessed duration of response (A), progressionfree survival (B), and overall survival (C)

All 101 patients assessable for activity in phase 2 are shown. The $\mathrm{x}$-axis shows time since infusion of chimeric antigen receptor $\mathrm{T}$ cells. $\mathrm{NE}=$ not estimable. 


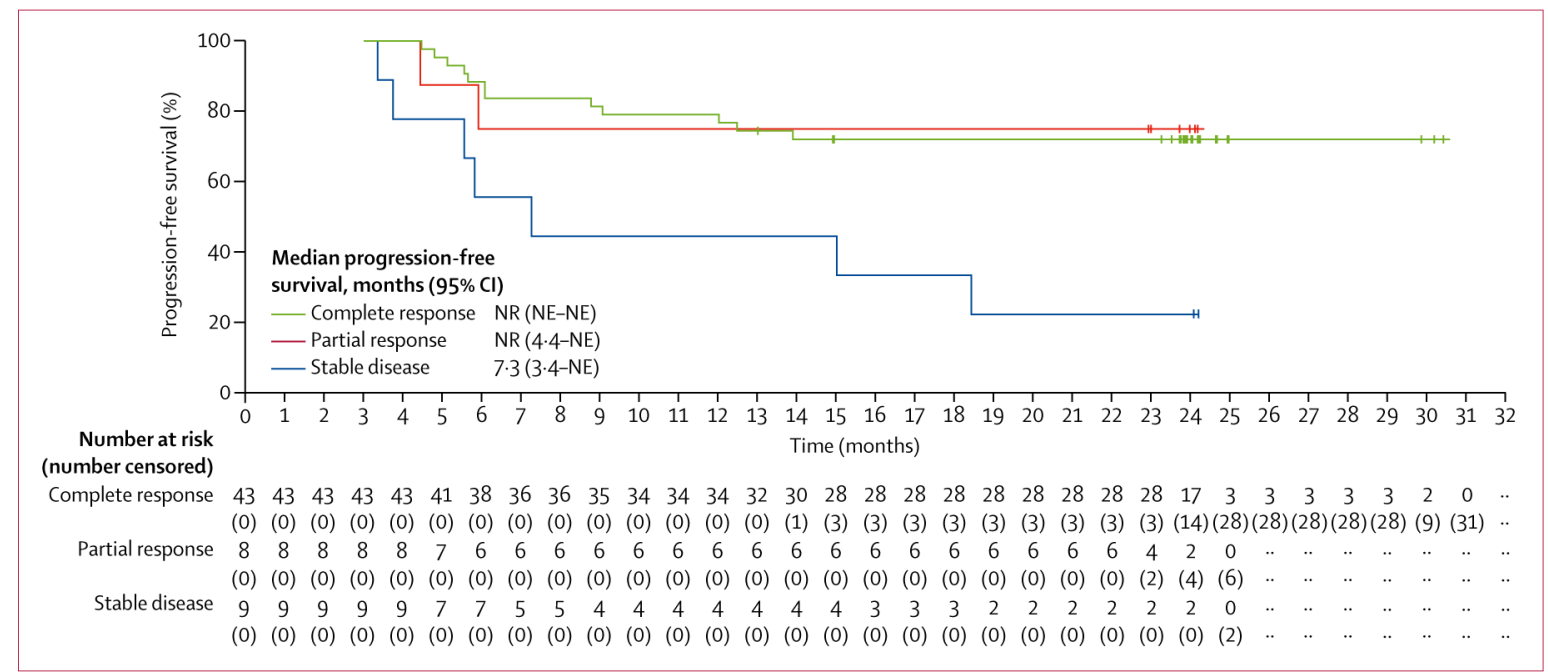

Figure 2: Post-hoc analysis of investigator-assessed progression-free survival by response status at 3 months after axicabtagene ciloleucel

60 patients with ongoing complete response, partial response, or stable disease month 3 in phase 2 are shown. The $\mathrm{X}$-axis shows time since infusion of chimeric antigen receptor $\mathrm{T}$ cells. Four of eight patients with partial responses and four of nine patients with stable disease at 3 months subsequently converted to complete responses. NR=not reached. $\mathrm{NE}=$ not estimable. 


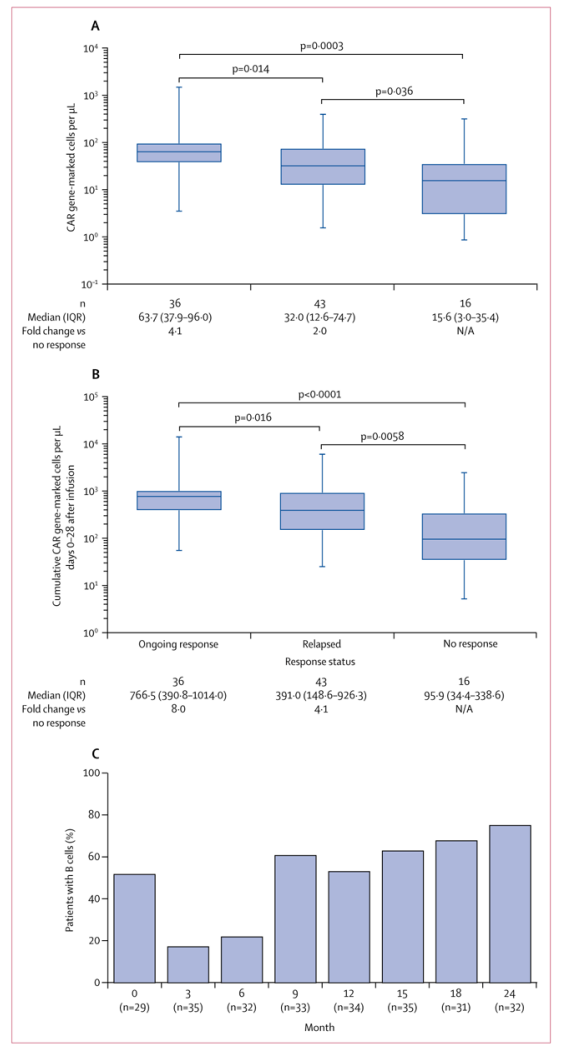

Figure 3: Post-hoc analysis of CAR T-cell and B-cell concentrations

CAR T cell exposure by peak concentrations of CAR T cells in peripheral blood (A) or area under the curve (B) and proportion of patients with responses at 24 months with detectable B cells (C). For (A) and (B), the horizontal line within each box represents the median, the lower and upper borders of each box represent the IQR, and the bars show the range. CAR area under the curve is defined as cumulative concentrations of CAR gene-marked cells per $\mu \mathrm{L}$ of blood during the first 28 days after axicabtagene ciloleucel therapy. For (A) and (B), these analyses include all 101 phase 2 patients assessable for activity with the exception of two patients who received subsequent anticancer therapy while in response to axicabtagene ciloleucel, two patients who died before disease progression, and two patients for whom CAR T-cell data were not available. For (C), this analysis includes patients in ongoing response with assessable samples and excludes two patients who received subsequent anticancer therapy while in response to axicabtagene ciloleucel. $\mathrm{CAR}=$ chimeric antigen receptor. $\mathrm{N} / \mathrm{A}=$ not applicable. 
Table 1:

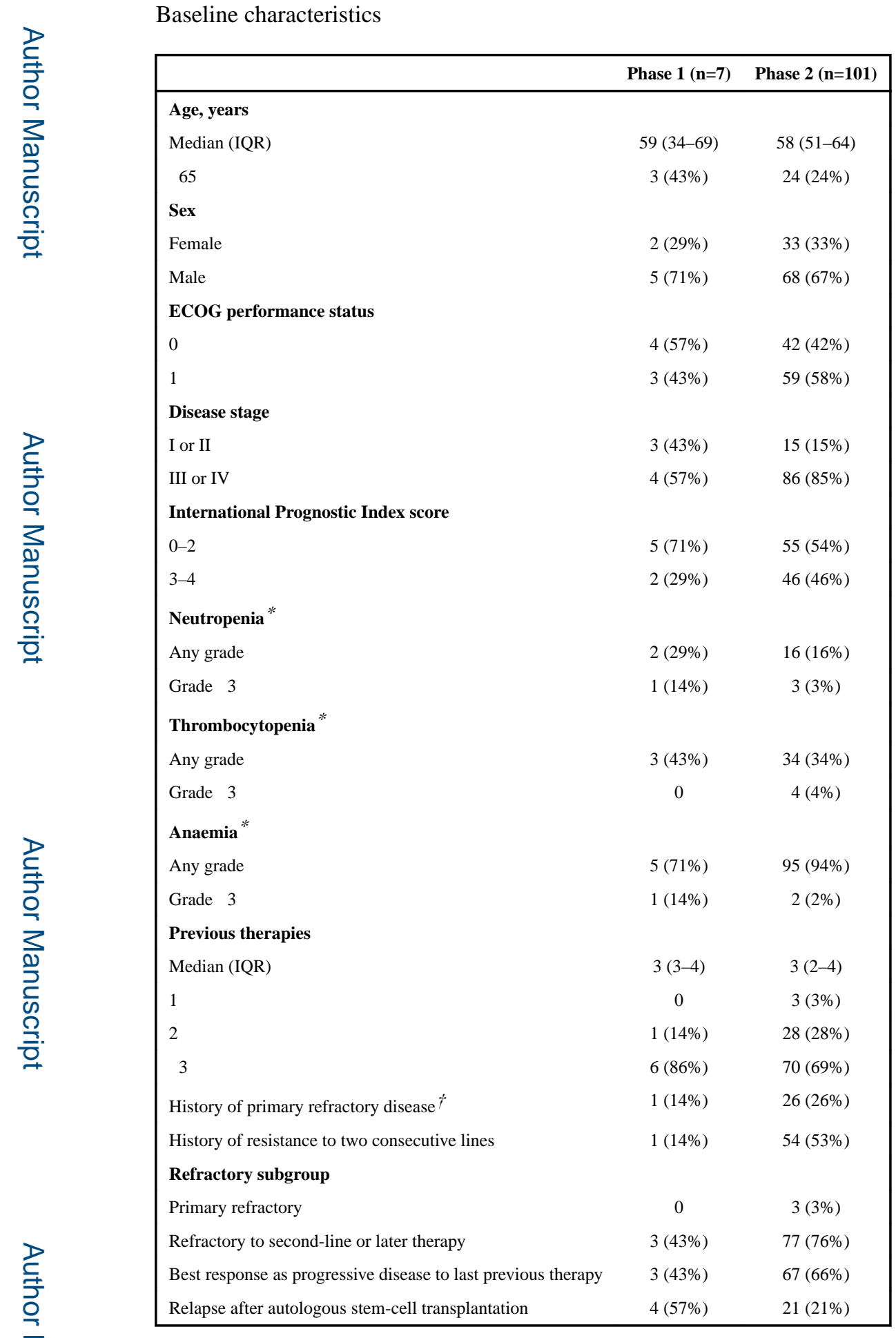

Data are $\mathrm{n}(\%)$, unless otherwise indicated. 
* Baseline neutropenia, thrombocytopenia, and anaemia were assessed according to laboratory values for decreased neutrophils, decreased platelets, and decreased haemoglobin, respectively, before conditioning chemotherapy according to the National Cancer Institute's Common Terminology Criteria for Adverse Events (version 4.03). ${ }^{18}$

${ }^{\dagger}$ Patients could have had other therapies after primary refractory disease. 
Table 2:

Investigator-assessed and IRC-assessed response in phase 2 participants

\begin{tabular}{|lcc|}
\hline & $\begin{array}{l}\text { Investigator-assessed } \\
(\mathbf{n = 1 0 1})\end{array}$ & $\begin{array}{l}\text { IRC-assessed } \\
(\mathbf{n = 1 0 1})\end{array}$ \\
\hline Objective response $^{*}$ & $84(83 \%)$ & $75(74 \%)$ \\
$\quad$ Complete response ${ }^{\dagger}$ & $59(58 \%)$ & $55(54 \%)$ \\
$\quad$ Partial response & $25(25 \%)$ & $20(20 \%)$ \\
Ongoing response ${ }^{*}$ & $39(39 \%)$ & $36(36 \%)$ \\
$\quad$ Complete response & $37(37 \%)$ & $35(35 \%)$ \\
$\quad$ Partial response & $2(2 \%)$ & $1(1 \%)$ \\
Median duration of response, months $(95 \% \mathrm{CI})$ & $11 \cdot 1(4 \cdot 2-\mathrm{NE})$ & $\mathrm{NR}(10 \cdot 9-\mathrm{NE})$ \\
Median duration of complete response, months $(95 \% \mathrm{CI})$ & $\mathrm{NR}(12 \cdot 9-\mathrm{NE})$ & $\mathrm{NR}(\mathrm{NE}-\mathrm{NE})$ \\
Median overall survival, months $(95 \% \mathrm{CI})$ & $\mathrm{NR}(12 \cdot 8-\mathrm{NE})$ & $\mathrm{NR}(12 \cdot 8-\mathrm{NE})$ \\
\hline
\end{tabular}

Data are $\mathrm{n}(\%)$, unless otherwise indicated. All 101 patients who received axicabtagene ciloleucel in phase 2 of ZUMA-1 are shown. IRC=independent central review committee. $\mathrm{NE}=$ not estimable. $\mathrm{NR}=$ not reached.

Concordance between investigators and the IRC for objective response was $81 \%(\kappa 0.45$ [95\% CI $0 \cdot 24-0 \cdot 65])$.

${ }^{\dagger}$ Concordance between investigators and IRC for complete response was 90\% ( $~ 0.80$ [95\% CI 0.68-0.92]).

${ }^{7}$ Three patients with ongoing response per investigator review were not ongoing responders per central review. Two of these patients underwent stem-cell transplantation before documented progression, which was considered a censor event per IRC but not per investigator assessment. The third patient was deemed to have progressive disease per IRC after 10.9 months but was assessed to be in ongoing response at 23.4 months per investigator assessment. 


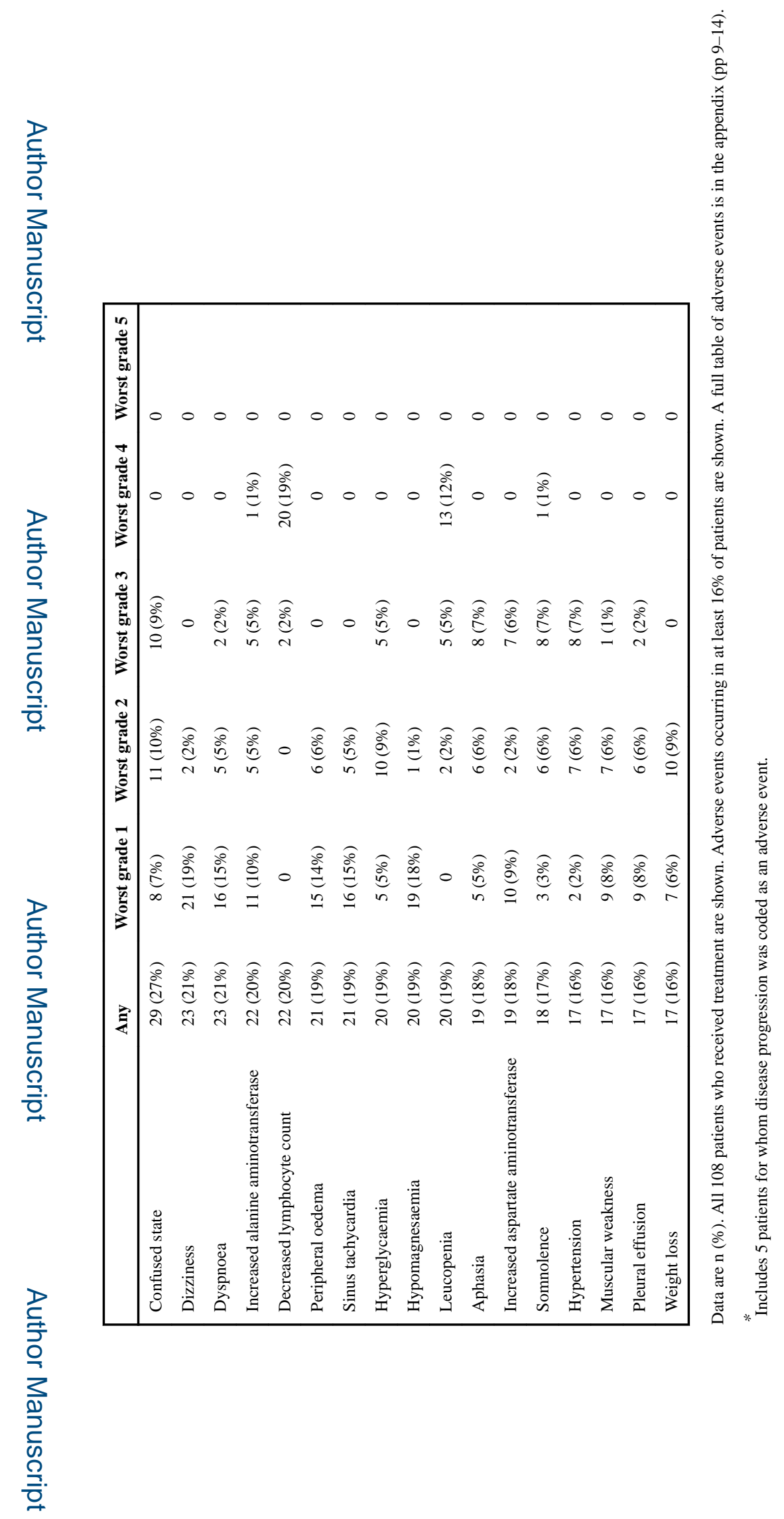

Lancet Oncol. Author manuscript; available in PMC 2019 September 09. 